\title{
A Causal Decision Making Model for Knowledge Management Capabilities to Innovation Performance in Taiwan's High-Tech Industry
}

\author{
Hung-Fan Chang', Gwo-Hshiung Tzeng²
}

\begin{abstract}
$R \& D$ and innovation is the source of technology companies' profit. If companies cannot be smoothly implemented technological innovation and R\&D investments, then they can not strengthen their competitiveness. In the light of dynamic capabilities and absorptive capacities, we need an effective multi-criteria decision-making (MCDM) analysis tools to assess the impact of organizational innovation and performance factors so that we can promote organizational innovation performance requires. This study used multiple criteria decision analysis method- DEMATEL, how influence as innovation performance on knowledge management capabilities of high-tech industry. Result of this study, enterprises can access external knowledge and make amendments to the operating principle effectively and immediately, when enhanced absorptive capabilities. Moreover, enhancing absorption capabilities will lead the improvement of dynamic capabilities to adapt to the rapidly changing competitive environment.
\end{abstract}

Keywords: Innovation Performance; Knowledge management capabilities; High-tech industry.

\footnotetext{
I Ph.D. student, Institute of Management of Technology, National Chiao Tung University. I00I University Road, Hsinchu City 300, Taiwan. E-mail: hfchang.mt98g@nctu.edu.tw TEL: +886-3-57/2121

${ }^{2}$ National Visiting Professor, Institute of Management of Technology, National Chiao Tung University. I00I University Road, Hsinchu City 300, Taiwan. E-mail: ghtzeng@cc.nctu.edu.tw TEL: +886-2-23।465। 5 ext 505
} 


\section{Introduction}

Technology The industrial structure is small and medium enterprises (SMEs) in Taiwan, it is high for most of companies using $5 \%$ innovation R\&D investment to total sales of turnover. According to 2006 statistics, the top ten technology companies in Taiwan (for example: TSMC, UMC, Foxconn, etc.) invested only $4.3 \%$ in R\&D on average. But compared to an average of $11.55 \%$ of the R\&D costs, which is Europe and the United States technology companies invested. What's more, Intel and Sun Microsystems invested as much as 17\% (Chesbrough, 2006). Domestic SMEs can not bear the high R\&D and innovation budget. With high risk and uncertainty characteristics of innovation activities, so that R\&D and innovation cannot be the core item in financial budget of the projects. R\&D and innovation is the source of technology companies' profit, if companies cannot be smoothly implemented technological innovation and R\&D investments, then they can not strengthen their competitiveness. Therefore, Taiwan's technology industry knowledge and management capability can be used to overcome the disadvantage of insufficient R\&D resources.

Teece et al. (1997) defines dynamic capabilities as firms' capabilities to integrate, the dynamic capabilities theory emphasizes that businesses should build internal and external information coordination to address rapidly changing environments. Thus, dynamic capabilities can be seen as a potential and emerging approach to capture the competitive advantages. Zahra and George (2002) further explained the absorptive capabilities from the perspective of dynamic capabilities, pointing out that the absorptive capabilities is a set of processes of analyzing knowledge accumulation and knowledge transformation, a competitive advantage is sustained and created through the development of dynamic capabilities. Thus, the absorptive capabilities enhance the corporate dynamic capabilities and the corporate dynamic capabilities enhance the organizational innovation performance. There are relations within the three. However, there are many criteria that dynamic capabilities and absorptive capacities would have impacts on organizational knowledge management. In the light of dynamic capabilities and absorptive capacities, we need an effective multi-criteria decision-making (MCDM) analysis tools to assess the impact of organizational innovation and performance factors so that we can improve organizational innovation performance requires.
In this study, we use decision making trial and evaluation laboratory (DEMATEL) mainly for the analysis on social science issues of relevant various factors, and to explore the issues or the causal relationship between factors. DEMATEL is a widely used tool for analyzing this type of problem. When a policy maker facing a number of criteria that needed to be improved, the best way is to find the factor that has the greatest impact on other criteria. Tzeng et al. (2007) study pointed out that DEMATEL face to assessment criteria affect each other, we must understand which is the main source of criteria before improving criteria and then overall satisfaction can be effectively improved. Therefore, the main advantage of DEMATEL is putting indirect relation into the cause and effect diagram, and it is an effective method to analyze the overall structure and system guidelines in a number of programs (Wu, 2008). Unlike the traditional multiple criteria decision making techniques which typically assume the criteria are mutually independent; this technique does not require this assumption but further helps the decision makers in identifying the casual relationships among criteria (Ou Yang et al., 2008). The DEMATEL provides a systematic approach in identifying criteria, the relationships, and the weights on which decisions we would like to carefully make. This study used multiple criteria decision analysis method- DEMATEL, how influence as innovation performance on knowledge management capabilities of high-tech industry in perspective of dynamic capabilities and absorptive capabilities.

This article is structured as follows. Section 2 presents the research model, questionnaires design and research objects. Section 3 presents the logic of DEMATEL and discusses the results. The final section presents conclusions, limitations and suggestions for future work.

\section{Method}

We decided to use expert survey research as an overall methodological approach for our empirical investigation. As suggested by a number of scholars, this is in fact a very powerful method for building a rich understanding of complex phenomena. This chapter is consists of research model, questionnaires design and research objects. 


\section{I Research Framework}

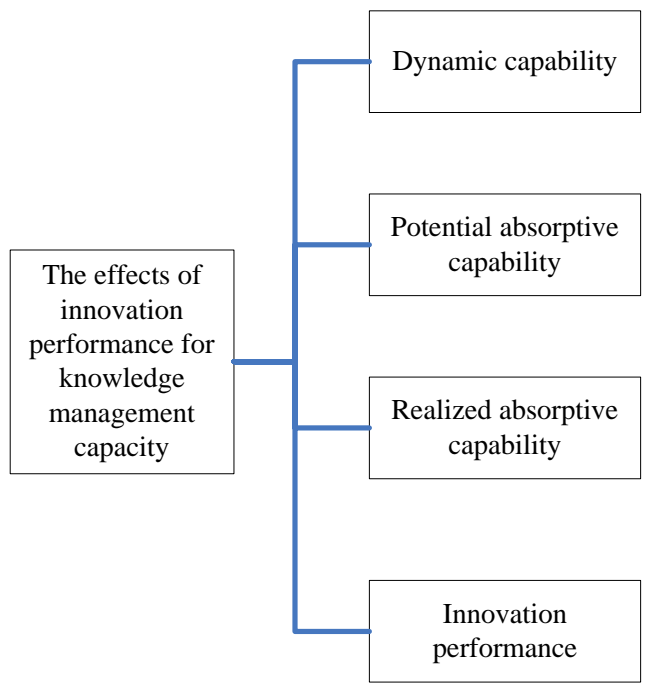

1.1 Flexible production system

1.2 Reengineering the degree of adaptation

1.3 Real-time adaptability

2.1 Technology transfer or licensing

2.2 External knowledge acquisition

3.1 The combination of external knowledge

3.2 Create new knowledge

5.1 New product development R\&D costs

$5.2 \mathrm{R} \& \mathrm{D}$ expenses/ total sales

5.3 New product development time
1.4 Integration capacity of the internal and external resources

1.5 Grasp the immediacy of information

2.3 Buffer time to adapt to new knowledge

2.4 Availability of external knowledge

3.3 Competitive advantage

3.4 Product features or transition processes

5.4 Process/ enhance the capacity utilization 5.5 Number of patents approved

5.6 The proportion of technology transfer

Figure I. Research framework of knowledge management capabilities

\section{Dynamic Capabilities}

Teece (1986) extends the dynamic capabilities concept from resource base, proposing that dynamic capabilities are the capabilities of change according to environmental changes. When the industrial environment changes rapidly and market competition is extremely fierce, dynamic capabilities emphasize the real-time response to the updating and reconfiguration of resource capabilities in order to adapt to environmental changes (Teece et al., 1997). Moreover, Teece (2007) stressed that the three stages of the implementation of dynamic capabilities the practical application of enterprise management systems: I. Echoing the views of Porter analysis of the competitive environment, identify threats to the external environment, competition; 2 . The use of organizational advantages, grasp the external opportunities; 3 . Integrate capabilities used of dynamic external resources within the organization.

Integration of the literature, all the dynamic capabilities emphasized business changes in response to external competitive environment adaptability. When the upgrading change rate of industry or technological, that the competitive environment will lead to a substantial increase in uncertainty.

\section{Knowledge Absorption Capabilities}

According to Cohen \& Levinthal (1990), acquired knowledge (for example basic skills, common languages etc.) will affect on firm's recognition of knowledge value, knowledge assimilation and usage. Hence, recognition of knowledge value, knowledge assimilation and capability of knowledge usage are called "absorptive capabilities" and is defined as the capability of a firm in recognition and assimilation of knowledge and the capability in using external knowledge.

Zahra and George (2002) who mentioned that potential absorptive capabilities include knowledge acquisition capabilities and knowledge assimilation capabilities, as well as efficient acquisition and application of external knowledge in order to improve effective knowledge transformation and application. And, Zahra and George (2002) using dynamic capabilities view of the flow reinterpretation of absorptive capabilities, that the absorptive capabilities is an analysis of organizational knowledge accumulation and flow of the flow through the dynamic capability to create and maintain the organization competitive advantage. 


\section{Innovation Performance}

The creation of innovation performance is consisted of a series of complicated innovation activities and those activities form a value chain. Damanpour et al. (2009) indicated that the impact organizational innovation performance depends on compositions of innovation types helps organizational capabilities and affects organizational conduct and results. KivimaÈki et al. (2000) argued that innovative performance assessed by indicators is perceived innovation effectiveness and technology innovation and intangible assets obtained. Chiesa et al. (2009) argued that measuring R\&D performance has become a fundamental concern for the issue has been evaluation the profitability of R\&D projects. Successful technology industries are not only generated innovative ideas, but also transfers these newly created business model through the organizational system for innovation performance (Thamhain, 2003). This paper focuses on the effects of organizational knowledge management and innovation performance on the competitiveness and market acceptance of the organization. In this paper, the innovation performance is measured with the three common criteria: I. product innovation; 2. process innovation; 3 . profitable innovation.

\subsection{Questionnaires Design}

In the questionnaire validity, that the test method is thought as well as nomological validity, and content validity. The questionnaire covered four dimensions which includes 19 questions: Potential absorptive capabilities (acquisition and assimilation) and realized absorptive capabilities (transformation and application) referring to Nonaka and Takeuchi (1995) and Quinn et al. (1999)'s knowledge absorptive capabilities evaluation. Dynamic Capabilities (integration and transformation) based on Nonaka and Konno (1998) and Wallin and Krogh (2010)'s dynamic capabilities studies and evaluation. And, innovation performance (product innovation, process innovation and profit innovation) based on Olson et al. (1995), Cooper \& Kleinschmidt (1996) and KivimaÈki et al. (2000)'s innovation performance measurement.

\subsection{Research Objects}

This study applying of expert surveys for technology industry's knowledge management influence innovation performance data, and factors influencing innovation performance correlation analysis. To ensure some homogeneity of work background, all subjects were selected from the 13 R\&D managers in Hsinchu Science Park. The primary criterion for selecting subjects was that they start a career over three years. They came from various companies on technology industries that include semiconductor fabrication plant and opto-electronics. The use of expert questionnaire survey to explore the impact of organizational factors on technology industries that the sample structure.

\section{KMC of Organization Empirical Studies}

The DEMATEL approach, used for studies and solving complicated, intertwined or MCDM problems, was successfully applied in many items, such as business strategies, marketing strategies, education evaluation, project planning and service quality (Tseng, 2009; Büyüközkan and Öztürkcan, 2010; Chen and Chen, 2010). Unlike the traditional multiple criteria decision making techniques which typically assume the criteria are mutually independent; this technique does not require this assumption but further helps the decision makers in identifying the casual relationships among criteria (Ou Yang et al., 2008).

We must understand which the main assessment criteria on DEMATEL. the main advantage of DEMATEL is putting indirect relation into the cause and effect diagram, and it is an effective method to analyze the overall structure and effect factors. DEMATEL can effectively understand the causal relationship, by matrix, "the overall dimensions" and the number of the causal impact strength. Gabus and Fontela (1973) pointed out that the DEMATEL three basic assumptions: I. A clear definition of the nature and characteristics; 2. To $0,1,2,3$ and 4 said the association between the strength of three criteria. Explain the relevance of various factors and management implications. This section is to present the results of DEMATEL, which can be summarized in the following steps:

\section{Step I: Find the Average Matrix.}

Suppose we have $H$ experts in this study and $n$ factors to consider. Each stakeholder is asked to indicate the degree to which he or she believes a factor $i$ affects factor $j$. These pairwise comparisons between any two factors are denoted by $a_{i j}$ and are given an integer score ranging from $0,1,2,3$, and 4, representing 'No influence (0),' 'Low influence (I),' 'Medium influence (2),' 'High influence (3),' 
and 'Very high influence (4),' respectively. The scores by each expert will give us a $n \times n$ non-negative answer matrix $\boldsymbol{X}^{k}=\left[x_{i j}^{k}\right]$, with $1 \leq k \leq H$. Thus $X^{1}, X^{2}, \ldots, X^{H}$ are the answer matrices for each of the $H$ experts, and each element of $X^{k}$ is an integer denoted by $x_{i j}^{k}$. The diagonal elements of each answer matrix $X^{k}$ are all set to zero. We can then compute the $n \times n$ average matrix $\boldsymbol{A}$ for all expert opinions by averaging the $H$ experts' scores as follows:

$$
a_{i j}=\frac{1}{H} \sum_{k=1}^{H} x_{i j}^{k}
$$

The average matrix $\boldsymbol{A}=\left[a_{i j}\right]$ is also called the initial direct relation matrix. $\boldsymbol{A}$ shows the initial direct effects that a factor exerts on and receives from other factors, shown as Table I Furthermore, we can map out the causal effect between each pair of factors in a system by drawing an influence map.

\begin{tabular}{cccccc}
\hline Dimensions & DC & PAC & RAC & IP & Total \\
\hline DC & 0.00 & 2.69 & 2.69 & 3.08 & 8.46 \\
PAC & 2.92 & 0.00 & 3.08 & 3.08 & 9.08 \\
RAC & 2.85 & 2.46 & 0.00 & 2.92 & 8.23 \\
IP & 2.85 & 2.46 & 2.31 & 0.00 & 7.62 \\
\hline Total & 8.62 & 7.62 & 8.08 & 9.08 & \\
\hline
\end{tabular}

Table I. The mean matrix A

\section{Step 2: Calculate the Normalized Initial Direct- Relation Matrix.}

The normalized initial direct-relation matrix $\boldsymbol{D}$ is obtained by normalizing the average matrix $\boldsymbol{A}$ in the following way:

$$
\begin{gathered}
\text { Let } s=\max \left(\max _{1 \leq i \leq n} \sum_{j=1}^{n} a_{i j}, \max _{1 \leq j \leq n} \sum_{i=1}^{n} a_{i j}\right) \\
\text { Then } D=\frac{A}{s}
\end{gathered}
$$

Since the sum of each row $j$ of matrix $\boldsymbol{A}$ represents the total direct effects that factor $i$ gives to the other factors, $\max _{1 \leq i \leq n} \sum_{j=1}^{n} a_{i j}$ represents the total direct effects of the factor with the most direct effects on others. Likewise, since the sum of each column $i$ of matrix $\boldsymbol{A}$ represents the total direct effects received by factor $i, \max _{1 \leq j \leq n} \sum_{i=1}^{n} a_{i j}$ represents the total direct effects received of the factor that receives the most direct effects from others. The positive scalar $s$ takes the lesser of the two as the upper bound, and the matrix $\boldsymbol{D}$ is obtained by dividing each element of $\boldsymbol{A}$ by the scalar s. Note that each element $d_{i j}$ of matrix $\boldsymbol{D}$ is between zero and less than I.

\begin{tabular}{cccccc}
\hline Dimensions & DC & PAC & RAC & IP & Total \\
\hline DC & 0.00 & 0.22 & 0.22 & 0.27 & 0.72 \\
PAC & 0.24 & 0.00 & 0.26 & 0.26 & 0.76 \\
RAC & 0.24 & 0.21 & 0.00 & 0.24 & 0.69 \\
IP & 0.24 & 0.21 & 0.19 & 0.00 & 0.63 \\
\hline Total & 0.72 & 0.63 & 0.67 & 0.77 & \\
\hline
\end{tabular}

Table 2. Direct-relation matrix D 


\begin{tabular}{ccccc}
\hline Dimensions & $\mathrm{r}+\mathrm{c}(\mathrm{a})$ & $\mathrm{r}-\mathrm{c}(\mathrm{b})$ & $(\mathrm{a})+(\mathrm{b})$ & Rank of impact \\
\hline DC & 0.72 & 0.72 & 1.44 & $\mathrm{I}$ \\
PAC & 0.76 & 0.63 & 1.39 & 3 \\
RAC & 0.69 & 0.67 & 1.36 & 4 \\
IP & 0.63 & 0.77 & 1.40 & 2 \\
\hline
\end{tabular}

Table 3 The level of direct impact

The direct-relation matrix (D) can be added to the ranks and the degree of importance of the direct-relation. Among them, the "potential absorptive capabilities" is the direct-relation matrix (D) is the most important of dimensions (rows+ columns= I.39); Second, the order of knowledge sharing, specific absorption, dynamic capabilities and innovative performance, as shown in Table 3.

\section{Step 3: Compute the Total Relation Matrix.}

A continuous decrease of the indirect effects of problems along the powers of matrix $\boldsymbol{D}$, e.g. $D^{2}, D^{3}, \ldots, D^{\infty}$, guarantees convergent solutions to the matrix inversion similar to an absorbing Markov chain matrix. Note that $\lim _{m \rightarrow \infty} \boldsymbol{D}^{m}=[0]_{n \times n} \quad$ and $\lim _{m \rightarrow \infty}\left(\boldsymbol{I}+\boldsymbol{D}+\boldsymbol{D}^{2}+\boldsymbol{D}^{3}+\ldots+\boldsymbol{D}^{m}\right)=(\boldsymbol{I}-\boldsymbol{D})^{-1}$, where 0 is the $n \times n$ null matrix and $I$ is the $n \times n$ identity matrix. The total relation matrix $T$ is an $n \times n$ matrix and is defined as follow:

$$
\boldsymbol{T}=\left[t_{i j}\right] \quad i, j=1,2, \ldots, n
$$

where $\boldsymbol{T}=\boldsymbol{D}+\boldsymbol{D}^{2}+\ldots+\mathbf{D}^{m}=$ $\boldsymbol{D}+\boldsymbol{D}^{2}+\ldots+\boldsymbol{D}^{\mathrm{m}}=\boldsymbol{D}\left(I+\boldsymbol{D}+\boldsymbol{D}^{2}+\ldots+\boldsymbol{D}^{m-1}\right)$

$$
=\boldsymbol{D}\left[\left(I+\boldsymbol{D}+\boldsymbol{D}^{2}+\ldots+\boldsymbol{D}^{m-1}\right)(1-\boldsymbol{D})\right](1-\boldsymbol{D})^{-1}=
$$

$\mathbf{D}(\mathbf{I}-\mathbf{D})^{-1}$, as $m \rightarrow \infty$

We also define $r$ and $c$ as $n \times I$ vectors representing the sum of rows and sum of columns of the total relation matrix $\boldsymbol{T}$ as follows:

$$
\boldsymbol{r}=\left[\mathfrak{J}_{i n \times 1}=\sum_{j=1}^{n} t_{i j}\right.
$$

$$
\boldsymbol{c}=\left[c_{j}\right]_{1 \times n}^{\prime}=\left(\sum_{i=1}^{n} t_{i j}\right)_{1 \times n}^{\prime}
$$

where superscript ' denotes transpose.

Let $r_{i}$ be the sum of $i$-th row in matrix $\mathbf{T}$. Then $r_{i}$ shows the total effects, both direct and indirect, given by factor $i$ to the other factors. Let $c_{j}$ denotes the sum of $j$-th column in matrix $\boldsymbol{T}$. Then $c_{j}$ shows the total effects, both direct and indirect, received by factor $j$ from the other factors. Thus when $j=i$, the sum $\left(r_{i f}+\right)$ gives us an index representing the total effects both given and received by factor $i$. In other words, $\left(r_{i f}+\right.$ ) shows the degree of importance (total sum of effects given and received) that factor $i$ plays in the system. In addition, the difference ( $r_{i f}-$ ) shows the net effect that factor $i$ contributes to the system. When $\left(r_{i f}-\right)$ is positive, factor $i$ is a net causer, and when ( $r_{i f}-$ ) is negative, factor $i$ is a net receiver (Tzeng et al. 2007; Tamura et al., 2002), shown as Table 4.

\section{Step 4: Set a Threshold Value and Obtain the Impact- Relations-Map.}

In order to explain the structural relation among the factors while keeping the complexity of the system to a manageable level, it is necessary to set a threshold value $p$ to filter out some negligible effects in matrix $\mathbf{T}$. While each factor of matrix $\boldsymbol{T}$ provides information on how one factor affects another, the decision-maker must set a threshold value in order to reduce the complexity of the structural relation model implicit in matrix $\boldsymbol{T}$. Only some factors, whose effect in matrix $\boldsymbol{T}$ is greater than the threshold value, should be chosen and shown in an impact-relationsmap (IRM) (Tzeng et al., 2007). In this paper, the threshold value has been decided by experts. As long as the threshold value has been decided, the final result can be shown in an IRM. 


\begin{tabular}{lcccccc}
\hline Dimensions & DC & PAC & RAC & IP & r+c & $r-c$ \\
\hline DC & 2.53 & $2.69 *$ & $2.74^{*}$ & $2.90^{*}$ & 27.01 & -0.05 \\
PAC & $2.86 *$ & $2.63 *$ & $2.90^{*}$ & $3.04 *$ & 27.56 & 0.77 \\
RAC & $2.74 *$ & $2.69 *$ & $2.58 *$ & $2.91 *$ & 27.27 & -0.12 \\
IP & $2.52 *$ & 2.48 & 2.52 & 2.48 & 26.83 & -2.00 \\
\hline
\end{tabular}

Note I : Bold is higher than the threshold value, the formation of self-related

Note $2 *$ That affect relationship is higher than the threshold value (2.58)

Table 4. The total-relation matrix for dimension

According to the results above, resultant vector $(r+c)$ higher, on behalf of the dimensions and other dimensions of the relationship between the greater impact (relevance). Vectorial difference $(r-c)$ on behalf of the net total impact matrix of the relationships (influence). If $r-c>0$, indicates that the dimensions of the degree of influence other dimensions than the affected level; however, $r-c<0$, indicates that the dimensions of the extent of impact of other dimensions than is the impact. In this study, the criteria of $r+c$, $r c$ show the relationship of the interaction in the impact-relations-map, shown in figure 2.

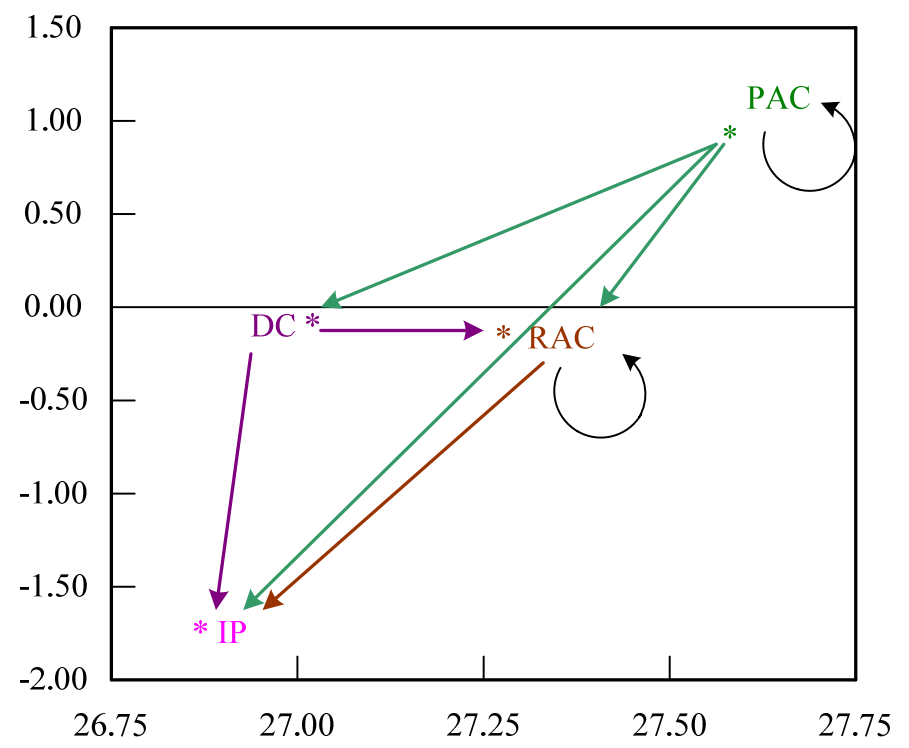

Figure 2. Figure of full direct/indirect influence matrix

The Innovation performance (IP) is the largest dimension to be affected, will be, potential absorptive capabilities (PAC), realized absorptive capabilities (RAC) and the dynamic capabilities (DC) are affected. PAC is the largest impact structure, emphasizing the internal information flow and the degree of interaction with each other, and to enhance knowledge sharing through knowledge acquisition and utilization.

PAC for the DC will have a positive impact. Zahra and George (2002) study pointed out that the PAC contains the company's knowledge acquisition capabilities and knowledge assimilation capabilities, and effective access to the external knowledge to business development process, can improve the R\&D performance. And Szulansli (1996) study pointed out that different industries or corporate culture, for knowledge acquisition capabilities, knowledge assimilation and knowledge of the values are different. Therefore, the factors will affect the PAC, not only include knowledge acquisition capabilities and knowledge assimilation capabilities, but also are related to the industry background and corporate culture. The surrounding literature on knowledge absorption capabilities appears to be generally compatible with the more detailed results 
obtained in the present study. The aim of PAC can enhance operation knowledge and technical capabilities on enterprise. The function of PAC is improving access to the ratio of external knowledge, which through knowledge transfers, licensing or buying patterns. Thus, PAC can reduce the use of external knowledge into new knowledge enterprises buffer time, improve development efficiency.

Zahra and George (2002) study pointed out that the RAC includes knowledge transformation capabilities and knowledge exploitation capabilities. And Christensen et al. (1998) for an urgent need for research and development of innovative technology companies, stressed that effective knowledge exploitation capabilities can help companies increase their R\&D efficiency. Therefore, these findings are in line with previous studies, the efficiency of RAC is emphasis knowledge integration between the enterprises of internal and external. Result of this part, dynamic capabilities is tied closely with knowledge creativity and $R \& D$ innovation performance after import external knowledge.

Teece et al. (1997) defined dynamic capabilities of enterprise integration, build and reconfigure internal and external capabilities to adapt to rapidly changing market environment. The study found, the function of $D C$ is knowledge integration and transformation to accord market demand, which organize, process and technology integration. Knowledge integration is the company introduced the use of leverage external resources. Transformation capabilities of enterprises is facing the sector restructuring, reorganization or staff mobility, the re-adjustment and adaptability, and help enterprises to face competition, changes in the environment of real-time response capabilities.

According to the results that, PAC is a mediating effect, while the DC and the RAC for the IP with the most positive and direct effect. Compile a comprehensive study of model results of the research the following three points: I. If we can improve corporate PAC can be effective access to external knowledge, to strengthen or amend their operational guidelines; 2 . RAC the upgrade, the upgrade will bring $D C$ to meet the rapidly changing competitive environment; 3 . Enterprises to adapt to changes in external competitive environment, capability to adapt to changes in magnitude when the industry or technology upgrading, the competitive environment will create hatred of increased uncertainty, and innovation performance of enterprises will be affected with DC.

\section{Conclusion}

The study concluded that knowledge management does not exist independently. It combines internal and external resources, and then to enhance the business, the organization sector or the industry competitiveness. However, the innovation performance is affected by the rising R\&D costs that cause lower earnings. Enterprises need an effective management to enhance the competitiveness. Result of this study, enterprises can access external knowledge and make amendments to the operating principle effectively and immediately, when enhanced absorptive capabilities. Moreover, enhancing absorption capabilities will lead the improvement of dynamic capabilities to adapt to the rapidly changing competitive environment.

To practical management implications, if the business upgrades the potential absorptive capabilities, it can access external knowledge and make amendments to the operating principle effectively and immediately. Upgrading knowledge absorption capabilities will lead the improvement of dynamic capabilities to adapt to the rapidly changing competitive environment. Innovation performance is affected by the rising R\&D costs that cause lower earnings. Organizational knowledge management starts from corporate R\&D which results in technological innovation and through R\&D management and intellectual property management to help effectively manage the internal resources.

\section{Limitation and Suggestion}

I remain perfectly aware that the validity of any empirical study is limited to the scope of discuss. These studies need to be treated circumspectly, as the results may reflect in part the way in which the data were collected. Despite the large number of participants and the variety of the task, the design of the present study is not without limitations. The research centers on an empirical study involving experts from Hsinchu Science Park. In addition, KMC is a creative and technology will translate into economic success of business model innovation. The faced of different industries require different business models, its management and innovation necessarily involves risks and 
profit from uncertainty. However, the faced with the social environment changes and changes in industrial structure, there are different levels of industrial innovative business model, how to integrate the resources for Taiwan's overall industrial resources can be more open? This is a follow-up study to explore more deeply the question.

\section{References}

BÜYÜKÖZKAN, G. and Öztürkcan, D. (2010). An integrated analytic approach for Six Sigma project selection. Expert Systems with Applications, 37(8), 58355847.

CHEN, J. K., Chen, I. S. (20I0). Using a novel conjunctive MCE approach based on DEMATEL, fuzzy ANP, and TOPSIS as an innovation support system for Taiwanese higher education. Expert Systems with Applications, 37(3), 198I-1990.

CHESBROUGH, H.W. (2006). Open Business Models: How to Thrive in the New Innovation Landscape. Harvard Business School Press, New York.

CHIESA, V., Frattini, F., Lazzarotti, V., Manzini, R. (2009). Performance measurement in R\&D: exploring the interplay between measurement objectives, dimensions of performance and contextual factors. R\&D Management, 39(5), 488-519.

CHRISTENSEN, C. M., Suarez, F. F., Utterback, J. M. (1998). Strategies for Survival in Fast Changing Industries. Management Science, 44(I2), S207-S220.

COHEN, W. N., Levinthal, D. A. (1990). Absorptive Capabilities: A New Perspective on Learning and Innovation. Administrative Science Quarterly, 35, I I 28-I I 52.

COOPER, R. G., Kleinschmidt, E. J. (1996). Winning business in product development: the critical success factors. Research-Technology Management, 39, 18-29.

DAMANPOUR, F., Walker, R. M., Avellaneda, C. N. (2009). Combinative Effects of Innovation Types and Organizational Performance: A Longitudinal Study of Service Organizations. Journal of Management Studies, 46(4), 1467-6486.
GABUS, A., Fontela, E. (1973). Perceptions of the world problematique: Communication procedure, communicating with those bearing collective responsibility (DEMATEL report I). Battelle Geneva Research Centre, Switzerland Geneva.

KIVIMAÈKI, M, LaÈ nsisalmi, H., Elovainio, M., HeikkilaÈ, A., LindstroÈm, K., Harisalo, R., SipilaĖ, K., Puolimatka, L. (2000). Communication as a determinant of organizational innovation. R\&D Management, 30(I), 33-42.

NONAKA, I., Takeuchi, H. (1995). The knowledge-Creating Company: How Japanese Companies Create the Dynamics of Innovation. Oxford University Press, New York.

NONAKA, I., Konno, N. (1998). The concept of "Ba": Building a foundation for knowledge creation. California Management Review, 40(3), 40-54.

OLSON, E.M., Walker, O.C., Ruekert, R.W. (1995). Organizing for effective new product development: the moderating role of product innovativeness. Journal of Marketing, I, 48-62.

OU YANG, Y. P., Shieh, H. M., Leu, J. D., Tzeng, G. H. (2008). A novel hybrid MCDM model combined with DEMATEL and ANP with applications. International Journal of Operations Research, 5(3), I-9.

QUINN, J. B. (1999). Strategic Outsourcing: Leveraging Knowledge Capabilities. Sloan Management Review, 40(4), 9-21.

SZULANSKI, G. (1996). Exporting Internal Stickness: Impediments to the Transfer of Best Practice within the Firm. Strategic Management Journal, 17, 27-33.

TEECE, D. J. (1986). Transaction Cost Economics and the Multinational Enterprise. Journal of Economic Behavior and Organization, 7, 21-45.

TEECE, D. J., Pisano, G., Shuen, A. (1997). Dynamic Capabilities and Strategic Management. Strategic Management Journal, 18(7), 509-533.

TEECE, D. J. (2007). Explicating Dynamic Capabilities: the Nature and Microfoundations of (Sustainable) Enterprise Performance. Strategic Management Journal, 28(13), 13191350. 
THAMHAIN, H. J. (2003). Managing innovative R\&D teams. R\&D Management, 33(3), 297-3II.

TSENG, M. L. (2009). Using the extension of DEMATEL to integrate hotel service quality perceptions into a causeeffect model in uncertainty. Expert Systems with Applications, 36(5), 9015-9023.

TZENG, G. H., Chiang, C. H., Li, C.W. (2007). Evaluating Intertwined Effects in e-Learning Programs: A Novel Hybrid MCDM Model Based on Factor Analysis and DEMATEL. Expert Systems with Applications, 32(4), 10281044.

WALLIN, M. W., Krogh, G. V. (2010). Organizing for Open Innovation: Focus on the Integration of Knowledge. Organizational Dynamics, in press.

WU, W. W. (2008). Choosing Knowledge Management Strategies by Using A Combined ANP and DEMATEL Approach. Expert Systems with Applications, 35(3), 828-835.

ZAHRA, S. A., George, G. (2002). Absorptive Capabilities: a Review, Reconceptualization, and Extension. Academy of Management Review, 27(2), 185-203. 